\title{
A Metamodel and Model-based Design Rule Checking DSL for Verification and Validation of Electronic Circuit Designs
}

\author{
Adrian Rumpold and Bernhard Bauer \\ Institute of Computer Science, University of Augsburg, Germany
}

\begin{abstract}
Keywords: Domain-specific Modeling, Model-based Analysis, Model-based Testing and Validation, Systems Engineering, Electronic Design Automation, Design Rule Checking.

Abstract: Development of embedded systems depends on both software and hardware design activities. Quality management of development artifacts is of crucial importance for the overall function and safety of the final product. This paper introduces a metamodel and model-based approach and accompanying domain-specific language for validation of electronic circuit designs. The solution does not depend on any particular electronic design automation (EDA) software, instead we propose a workflow that is integrated into a modular, general-purpose model analysis framework. The paper illustrates both the underlying concepts as well as possible application scenarios for the developed validation domain-specific language, MBDRC (Model-Based Design Rule Checking). It also discusses fields for further research and transfer of the initial results.
\end{abstract}

\section{INTRODUCTION}

Embedded hardware and computing have become pervasive aspects of modern technology, which we encounter under a variety of different names: cyber-physical systems, embedded systems, smart devices, and not least the Internet of Things. While different in their concrete use cases and implementation, they all share a common underlying concept, the close connection between software and hardware aspects. Their design and development activities differ, but ultimately both need to be actively quality managed in order to attain a sufficient level of quality. This quality may be expressed in terms of freedom from errors, but may also refer to aspects such as reliability, dependability, and safety, depending on the application domain.

Hardware design, and the design of electronic circuits in particular, differs significantly from the activities in a software development process, since it touches on both the abstract design of electronic circuits as well as their physical embodiment in the form of printed-circuit boards (PCB), assemblies, and higher integration levels. Nonetheless, both domains rely on tool support to identify mistakes by the developer, enforce design rules, or validate certain properties of the system under development. Electronic design automation (EDA) tools commonly include such functionality, but only offer it as part of a manual workflow within the software package - mostly only available as non free, proprietary software with the risk of vendor lock-in.

This paper proposes a model-based solution to the challenge of automatic validation of electronic design rules, outside of a particular EDA tool suite. As a prerequisite, we introduce an Ecore-based metamodel for electronic circuits and illustrate the transformation from industry-standard textual description formats into this model representation. Based on this modeling approach, we then introduce $M B D R C$, a textual domain-specific language for description of design rules for electronic circuits represented as instances of the EDA metamodel, demonstrate its applicability and describe further research opportunities.

A caveat: Similar approaches are also found using similar terminology - in the domain of VLSI (very large scale integration) design of integrated circuits on the silicon level - however, this paper is not aimed at these applications but rather a component-level view of embedded hardware.

\section{Outline}

Section 2 discusses related work, regarding both the domain of electronic design automation and the metamodeling and analysis approaches shown in this paper. We introduce a metamodel for 
electronic circuits based on the KiCad open-source EDA software in section 3. Based on this modeling approach, we develop $M B D R C$, a textual domain-specific language and an associated analysis workflow for validation of electronic design rules in section 4. This section also demonstrates the applicability of our proposed approach in two realistic use cases. Section 5 summarizes the key results of this paper and suggests starting points for further research beyond these initial efforts.

\section{RELATED WORK}

\section{Metamodeling}

The de-facto standard for simulation of electrical circuits is the SPICE tool (Nagel, 1975; Quarles et al., 1993). SPICE uses a textual format for description of circuits on a component level, their electrical connections, and simulation parameters. The structural description forms a netlist, which lists instances of components, their terminals or pins (external connection points), and nets (the electrical connections between these terminals). A similar netlist format lies at the heart of our metamodel used for circuit description, introduced in section 3 .

Although it focuses on a chip-level view, the work by Fischbach et al. (2014) proposes an abstract, highly generic metamodel for netlists, also loosely based on the SPICE format.

In the automotive domain, the AUTOSAR standard (Automotive Open System Architecture) provides a metamodel specification for the description of ECU (electronic control unit) hardware resources (AUTOSAR, 2017). Using this metamodel, electronic components commonly found in the automotive field can be described on a hardware element level, with pins and pin groups forming their connection. While the standard allows for hierarchical nesting of hardware elements, the metamodel is not well suited for capturing component-level design models of hardware assemblies. Rather, it addresses a higher level of abstraction, and as such can be regarded more of a complement to the metamodel proposed in this work, rather than a substitute.

Previous effort has been directed at standardization of a common format for exchange of electronic designs, leading to the creation of the Electronic Design Interchange Format (EDIF, see IEC 61690-2:2000). This interchange format mostly covers lower-level aspects of VLSI design (very large scale integration). As such, it is not suited well for describing circuits on a component level. Nonetheless, its modeling approaches for schematics can also be applied in the context of this paper.

\section{Electrical and Design Rule Checking}

While current research in electrical engineering has shifted towards low-level validation of integrated circuit designs, related academic literature exists that applies to the checking of electrical and design rules on a circuit level:

Pelz (1992) proposes a general set-based interpreted approach for design-rule and design-for-testability checking, which transforms netlists (specified in the common SPICE format) into an equivalent abstract set representation. Validations are subsequently performed by interpretation of a domain-specific language over this set representation. Their work is primarily focused on VLSI design, nevertheless it serves as a fundamental example of the use of domain-specific languages for the validation of EDA designs. The approach provides sufficient expressiveness, however it requires significant mental effort for construction and validation of the actual validation scripts.

Furthermore, commercial EDA software packages include a variety of built-in design rules, on which this paper draws to derive realistic application scenarios for the developed validation language.

\section{Model Analysis}

Rumpold et al. (2017); Pröll et al. (2018) have previously introduced a conceptual and tooling framework for complex model-based analyses with applications across various domains. They describe a modular architecture based on domain-specific modeling languages and associated analyses, which can be combined into arbitrary analysis workflows. We expand upon this framework in this paper, by adding support for models from the EDA domain and implementing an accompanying analysis wrapper for the MBDRC validation language.

\section{A METAMODEL FOR ELECTRONIC CIRCUITS}

In this section, we describe a metamodel that allows capturing information about the structure of electronic circuits, metadata associated with them, as well as a facility for modeling libraries of electronic parts used during circuit design. 


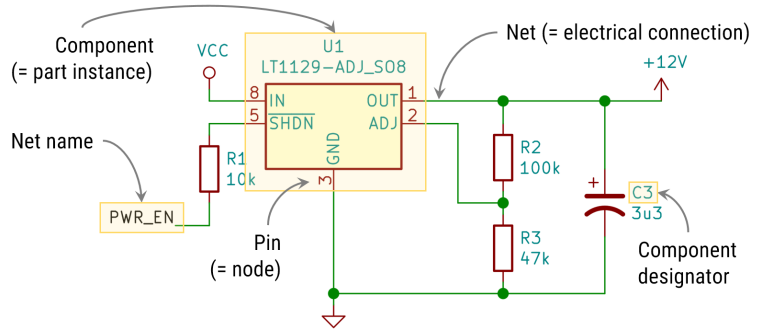

Figure 1: Terminology for common schematic elements.

\subsection{Motivation and Foundations}

Our metamodel closely follows the representation used by the popular open-source electronic design automation (EDA) software suite $\mathrm{KiCad}^{1}$. Chapters 11 and 15 of the official Eeschema (the schematic layout component of $\mathrm{KiCad}$ ) documentation describe the generation of netlists and the file format used by $\mathrm{KiCad}$ in greater detail (Charras and Tappero, 2018). The reasons for choosing this format as the basis for the EDA metamodel are twofold: First, the free and open-source nature of the $\mathrm{KiCad}$ tool suite ensures that the schematic capture software is universally available. Secondly, the format combines two concepts that benefit from a close connection, the modeling of the actual schematic as well as the abstract parts underlying the design. Similar textual representations exist for virtually all EDA software packages, the OrCAD Capture User Guide includes a comprehensive overview (Cadence Design Systems, 2016, ch. 20).

Conceptually, a schematic of an electronic circuit denotes the component symbols for the parts of the circuit and electrical connections between them. These connections between components form the basis for netlists, which list all nets formed by electrically connected parts (or their connection points, such as pins or pads on an integrated circuit). Figure 1 illustrates how these concepts relate to the graphical representation of circuit diagrams. From this structural description of electronic circuits the EMF metamodel for netlists shown in fig. 2 was derived.

EDA tools frequently include a library of common components, including their schematic symbols and information about physical properties such as pin assignments and their package. These libraries can also be extended by the designer, to accommodate for specific parts not available in the generic library.

Figure 3 shows our metamodel for such component libraries. Parts are grouped inside uniquely identified libraries and carry information

\footnotetext{
${ }^{1}$ http://www.kicad-pcb.org
}

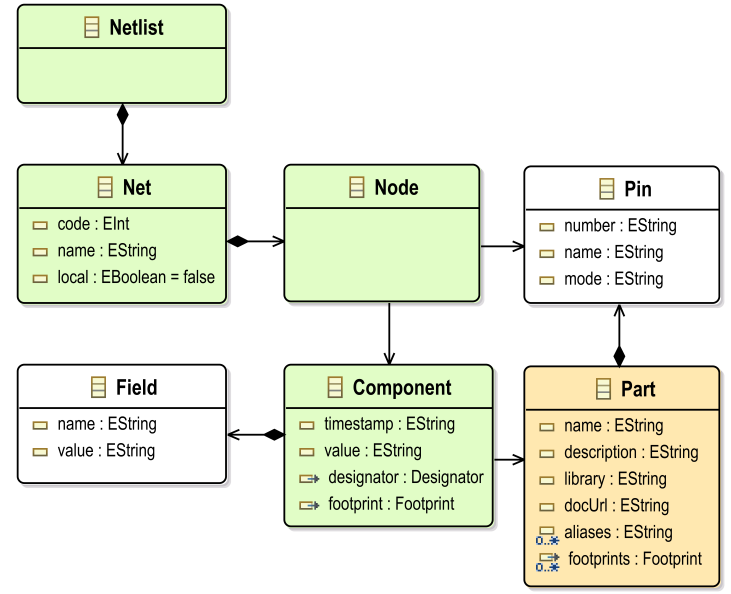

Figure 2: Metamodel for netlists.

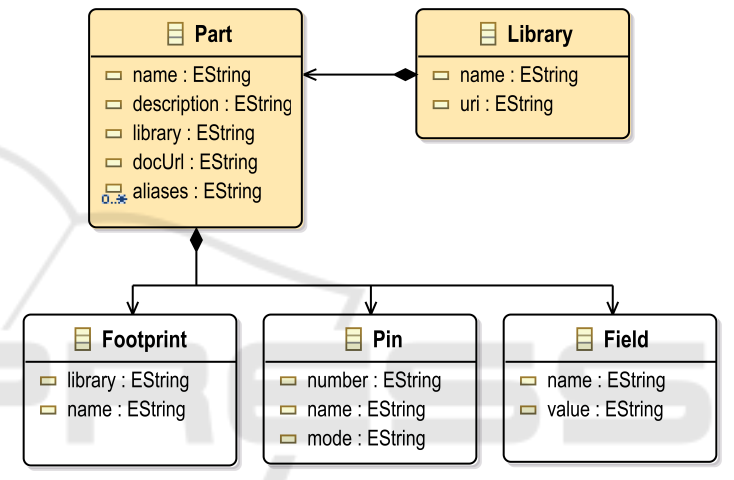

Figure 3: Metamodel for electronic component part libraries.

about their physical package and pin assignment, as well as textual fields stored as key-value pairs.

These key-value pairs can convey arbitrary additional information about a part or a specific component instance in a circuit, such as manufacturer part numbers, links to supplementary documentation, or simulation models and parameters.

The EDA metamodel has been defined as an instance of the EMF Ecore meta-metamodel. An accompanying parser allows to directly load file-based netlist representations as created by the KiCad software. This parser transforms the textual netlist into a proper instance of the EDA metamodel, which can be supplied as an input to the analysis framework introduced in the next section.

\subsection{Description of Metamodel Elements}

Part. A template element that abstractly describes a single electronic part and its basic properties (name and description, as well as alternative names [aliases]). 
Library In order to improve usability, EDA tools commonly group related parts into libraries, e.g. by function or manufacturer.

Footprint. In order to produce a printed circuit board (PCB) from a schematic, all components need to be assigned a footprint, which describes the physical packaging of the part. Since a single part may be offered in a variety of packages by its manufacturer, a single Part model element can be associated with multiple Footprint element instances, whereas only a single footprint is permitted for a concrete component in a circuit.

Pin. A single external connection point for a part, identified by its ordinal number with respect to the footprint of the part. A pin is further characterized by its mode of operation, e.g. output, input, or power supply pins of a part. Each part may contain any number of pins, although most electrical components contain at least two pins (notable exceptions are e.g. test points used for quality assurance and debugging purposes, which only contain a single connection point).

Field. Both library parts as well as components can carry arbitrary metadata in the form of key-value pairs, which may be used to convey additional information about the underlying circuit element (such as documentation references or procurement information).

Netlists. The collection of all nets in a circuit.

Net. An electric connection between one or more nodes. Nets are identified by a numeric code and may be assigned a unique name. In a hierarchical schematic, nets may also be designated as local, i.e. not visible outside the current hierarchy level.

Node. A node is the point where a single pin of a component makes connection with a net.

Component. A concrete instance of a library part on a circuit diagram. A component is identified by its reference designator on the schematic, usually comprised of a single-letter prefix and a numeric counter (e.g. C12 for a capacitor; see IEEE Std 315-1975 for comprehensive reference). Components are assigned a value, which further characterizes the component (e.g. the resistance or capacitance values for passive elements, or the concrete part name for a library part with multiple aliases). A unique timestamp allows to accurately match components, even when their designator changes, e.g. when the schematic is formatted in a different layout and designators are re-numbered.

\subsection{Use Cases}

The EDA metamodel described in this section forms the basis for the MBDRC validation language introduced below. However, other use cases besides circuit validation are possible, for example:

BOM Generation. Since the netlist model contains information about all components in a circuit, as well as additional metadata, a bill of materials (BOM) can be generated from it. The BOM lists all parts in a circuit, their designators, values, as well as additional procurement and/or assembly information. Identical parts can be grouped together to improve readability and conserve space.

The generation of a BOM is one example for the class of model-to-text (M2T) transformations that is possible using the EDA model as input.

Model Versioning. Given a representation of an electronic circuit as a model at different points in time, analysis of the evolution of the system over time in a semantic manner becomes possible (see Selic (2003, p. 23)). A persistent storage of these models allows to compare circuits between arbitrary revisions based on changes in the model elements, similar to approaches already found in the software modeling domain.

Furthermore, these snapshots can serve as baselines, from which different design variants can be derived; for example to evaluate different implementation options. Since our metamodel follows the Ecore meta-metamodel and is based on the set of Eclipse EMF technologies, model versioning approaches could be easily constructed using the EMF Compare ${ }^{2}$ feature (see also Brun and Pierantonio, 2008).

\section{MBDRC - A DSL FOR MODEL-BASED DESIGN RULE CHECKING}

\subsection{Foundations}

This section introduces a textual domain-specific language - MBDRC - for validation of electronic circuits based on the metamodel described above. Its name stems from the design rule checking (DRC) activities that form an integral part of the design of electronic circuits. Conventionally, such checks are

\footnotetext{
${ }^{2} \mathrm{https}: / /$ www.eclipse.org/emf/compare
} 
directly integrated into EDA tool packages, with little room for customization or secondary use outside the design tool.

We propose to separate the validation of design rules from the actual EDA tool used to design the system. This split enables use cases beyond the classical support of circuit designers in their daily work: For example, a standalone design validation can provide automated quality assessments of electronic designs in a similar fashion to state-of-the-art software engineering practice in the field of Continuous Integration. Analysis results can be visualized as part of a product quality dashboard, enabling a high-level overview of a system's development progress.

Furthermore, a stand-alone validation approach based on open technologies can help to alleviate the effects of vendor lock-in caused by the use of proprietary software solutions.

\subsection{Language Definition and Elements}

The MBDRC language is a textual domain-specific language defined using the Eclipse Xtext language engineering toolkit. ${ }^{3}$ It utilizes the EDA metamodel from the previous section to express rules used to assess the validity of a given electrical circuit design.

Overall Structure. As the top-level entity, an MBDRC script file may contain an arbitrary number of named rules. These rules provide a semantic grouping for validation expressions, against which a given netlist should be validated.

Validation expressions comprise a number of first-order logic expressions over the elements and attributes of the EDA metamodel introduced in the previous section.

Validation Expressions. Rules are composed of one or multiple quantified first-order predicates, denoted by the forall and exists keywords in the MBDRC language (corresponding to the $\forall$ and $\exists$ quantifiers). If a rule contains more than one quantifier, the overall rule is considered the logical conjunction of these quantified expressions.

Every quantified expression may reference an arbitrary number of target variables, which will be bound during evaluation by the interpreter. The list of target variables immediately follows the quantifier keyword (see the following section for concrete examples of the syntax). Each variable is associated with a type, either component, pin, or net, and must have

\footnotetext{
${ }^{3}$ https://www.eclipse.org/Xtext
}

a unique identifier. These variable definitions are collected in the sets $C, N, P$ or components, pins, and nets for each quantified expression.

Individual quantified expressions are evaluated by binding the quantified variables to all combinations of components, nets, and pins of the netlist. All constraints in the quantified expression are then evaluated using this valuation; if multiple constraints are specified, the overall result is obtained as their logical conjunction.

The actual constraints are propositional logic formulae (using $\vee, \wedge, \rightarrow, \neg$ ), with support for equality and inequality comparisons $(<,>, \leq, \geq,=, \neq)$, as well as property expressions on target variables, function calls, and array types.

Validation expressions can be scoped to only specific subsets of a netlist by specifying a where expression. During evaluation, only variable assignments that fulfill the scoping condition will be further validated against the rule body. By selecting appropriate scope conditions, the rule body can be simplified in order to improve readability and rule evaluation performance. Besides this main function of narrowing the scope for the rule body, the where clause also allows for a traversal of model structure, in a similar fashion to joins in SQL. This feature is further described in the next subsection.

Type Checking. Expressions in the MBDRC language are strongly typed and continuously type checked during the development of the script inside the editor as well as during the interpretation of the script.

The language supports both primitive types (boolean, integers, strings), complex types (as defined by the EDA metamodel), as well as sets of these.

Function expressions are statically typed, i.e. their parameter and return values types must be known at design time. Overloads of different return types are not currently supported in the DSL.

\subsection{Rule Script Execution}

The MBDRC domain-specific language forms the basis for an automated validation of a given netlist against a set of design rules. While in theory the analysis could be performed inside standalone validation tool, we envision its use in a more complex workflow. Therefore, the MBDRC analysis has been integrated into the model-based analysis framework previously described in Rumpold et al. (2017); Pröll et al. (2018). The addition of the EDA metamodel proposed in the previous section adds a new modeling domain to the set of domain specific modeling 


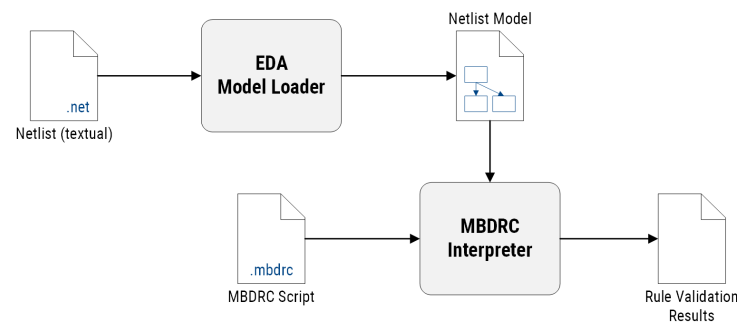

Figure 4: Execution workflow for an MBDRC analysis.

languages already included in the base framework (see Rumpold et al. (2017, fig. 1) for details).

The MBDRC analysis is available as one analysis unit along the execution graph to be processed by the analysis framework. It depends on a previous loader step, which transforms the textual representation of the input netlist into a proper domain model, and produces one or multiple analysis result objects. These describe the success or failure of each MBDRC rule that was validated, including detailed information about rule violations. Figure 4 illustrates the flow of information and dependencies between the steps of MBDRC validation.

The results can then be further processed, depending on the use case for the analysis: In an interactive setting, it might be desirable to highlight each circuit element that violates any design rules to aid the designer in fixing these problems. If the analysis is used to produce a quality report document, a more coarse-grained listing of all passed or failed design rules can be generated, omitting information about individual component-level elements. Generating such reports can be delegated to downstream model-to-text steps in the execution flow, to promote a proper separation of concerns between analysis modules.

\subsection{Application Examples}

The following section aims to illustrate the capabilities of the MBDRC language by applying it to two common tasks found in the EDA design process.

This selection of examples in this paper is by no means exhaustive, but rather seeks to provide an overview of the language elements and their possible applications.

\section{Validation of Documentation and Manufacturing Information}

Besides the structural information, the EDA metamodel introduced in the previous section also allows to attach metadata to each component in a circuit, or to the underlying library part, in the form of arbitrary key-value fields.

One use case for this information is to verify the availability of parts from a distributor and monitor the life cycle status of critical components as part of obsolescence management (see IEC 62402:2007 as an example for a relevant international standard).

The following MBDRC code snippet enforces that each component must be annotated with a distributor or manufacturer part number, unless it is actively marked as not being a critical part for the BOM (bill of materials):

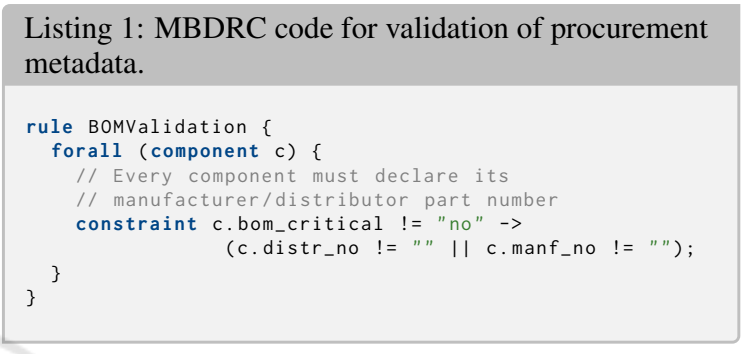

Listing 1 illustrates the basic structure of an MBDRC script: Each validation rule, identified by an arbitrary name, may contain one or more quantified expressions to specify the target model elements. Each quantified validation expression defines constraints to be verified against matching elements from the netlist model under test. Both block and single-line comments may be added to the script in a syntax familiar from the $\mathrm{C}$ or Java programming languages.

\section{Electrical Rules Checking}

As a more comprehensive demonstration of the capabilities of the MBDRC language, this example shows the implementation of simple electric rules checks as a composite MBDRC rule. These checks are commonly found in EDA tools to prevent logical errors during design of an electronic circuit and serve to validate the design during the schematic capture phase of the product life-cycle.

ERC rules for example guarantee that no two power sources are directly connected, or that components are not shorted out (which effectively renders them useless in the circuit, since they are bypassed). Most commercial EDA packages include a variant of this check; fig. 5 depicts the configuration of the pin compatibility matrix in the KiCad ERC checker as one representative example.

Listing 2 shows the implementation of a subset of these checks in the MBDRC language. The example demonstrates some of the more advanced features of the DSL: 
- Multiple quantified expressions can be combined to form composite rules. The overall rule evaluation result is formed by taking the logical conjunction of each quantified expression in the rule.

- where $(p 1$.net $==p 2$.net $)$ Scope filters can selectively apply constraints to model elements matching a filter expression. Complex property expressions (references to other model elements; in this example the net associated to a single pin) allow for comfortable model traversal similar to joins in relational databases.

- p2.mode in ["output", "power_out", "3state"]: The language supports sets of primitive types and membership testing for element properties, as a syntactical shorthand to improve readability.

- $\operatorname{card}(\mathrm{p}$. nets $)<=1: \quad$ Function expressions allow computation of values from model properties (the example determines the number of nets a pin is connected to as the cardinality of the set). The set of available functions is currently fixed, future research may add the possibility of declaring additional functions as part of the MBDRC language.

- not exists: In order to improve readability, the result of quantified expressions may be negated. This does not change the expressiveness of the language, since the negation might also be pushed inside the expression (compare first-order logic: $\neg \exists x . P(x) \Leftrightarrow \forall x . \neg P(x))$

- severity=info and message "Pin mode unspecified": Rules may specify a custom level of severity (info, warning, error) as well as a informative message to be displayed to the user, if the rule is found to be violated during evaluation.

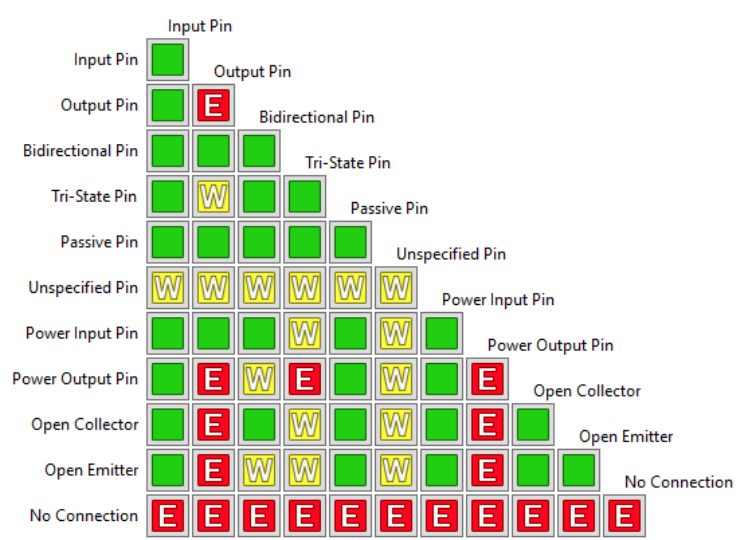

Figure 5: ERC pin compatibility validation rules provided by KiCad.

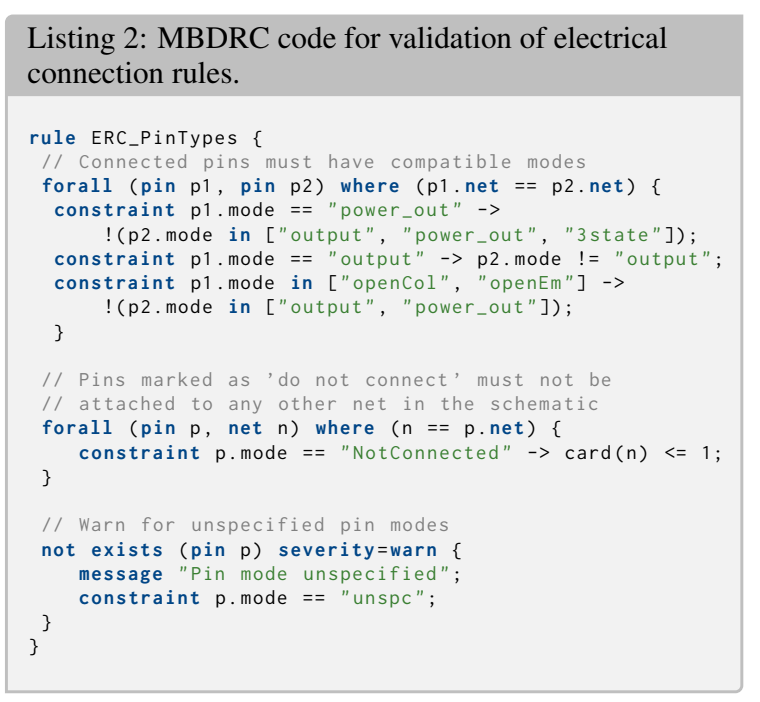

\section{CONCLUSION}

This paper has introduced two key results: First, we have proposed a general-purpose metamodel for electronic circuits derived from an industry-standard netlist representation. The metamodel allows capturing structural information about electronic circuits, as well as metadata and library information about the parts used in these circuits.

Subsequently, we have described a textual domain-specific language for the analysis and validation of circuits represented as instances of this metamodel. Several application examples demonstrate the language features as well as the DSL's suitability as a complement to the validation functionality found in common EDA tools.

We foresee that our approach can supplement the established design workflow for electronic circuits, by uncoupling the checking of design and electrical rules from any concrete EDA software suite. This additional freedom allows for easier tool interoperability and enables new use cases for these analyses.

\section{Future Work}

Based on our preliminary research introduced in this work, we envision a number of possible scenarios for further research, expanding the scope both towards more abstract system-level views, as well as sub-circuit level design activities.

Generation of Validation Code. The first prototype of the MBDRC DSL uses a separate 
parser to validate the rules defined in an MBDRC script against a concrete netlist. This approach facilitates the rapid co-evolution of the language syntax and its associated semantics, but does not fully utilize the power of the model-based approach. Instead, validation code for a given set of rules can be generated directly. The set of technologies for implementation of the first prototype of the DSL was chosen with this extension in mind: Xtext offers rich support for generating Java (among other languages) code from a DSL script (see Bettini, 2016, ch. 5).

Physical Layout Validation. While the current implementation of the MBDRC language is based on the metamodel for the abstract circuit representation embodied by netlists, the same concepts hold for the validation of physical circuit layouts. Here, the validation focuses on the positioning and electrical connections between components on a printed circuit board (PCB). Common questions during PCB design revolve around minimum clearance between adjacent tracks on the board, physical dimensions of components, or violations of manufacturing process capabilities (e.g. minimum drill sizes for holes or minimum track widths that can be manufactured).

The EDA metamodel can be extended to also include the physical positioning of components on a printed circuit board, the tracks that correspond to nets, as well as the physical dimensions of the board and the components to be placed on it. By adding appropriate mathematical operations to the MBDRC language, it can then be used to answer these physical design questions.

Test Plan Generation. Our last suggestion for further research focuses on the test of hardware components. Based on the model representation, as well as higher-level descriptions of requirements and associated test goals, we envision a strategy for planning of testing activities: By establishing a model link between requirements, test goals, as well as the actual components under test in a single integrated model, test cases may be derived that fulfill these test goals. One example might be the goal of verifying the correct function of fault tolerance mechanisms by means of fault injection. The circuit model provides the necessary information about the available signals as well as potentially affected components, while the traceability of the model allows to identify affected software components. The integrated view on both these domains allows for a clearer picture of the dependencies between components, as well as the necessary development steps in order to achieve certain test goals.

\section{REFERENCES}

AUTOSAR (2017). Specification of ECU Resource Template. Specification 060.

Bettini, L. (2016). Implementing Domain-Specific Languages with Xtext and Xtend. Packt Publishing, Birmingham, Mumbai, 2nd edition.

Brun, C. and Pierantonio, A. (2008). Model Differences in the Eclipse Modelling Framework. CEPIS UPGRADE, IX(2):29-34.

Cadence Design Systems (2016). OrCAD Capture User Guide. Technical Documentation.

Charras, J.-P. and Tappero, F. (2018). Eeschema Reference Manual. http://docs.kicad-pcb.org/master/ en/eeschema.html.

Fischbach, R., Heinig, A., and Schneider, P. (2014). Design rule check and layout versus schematic for $3 \mathrm{D}$ integration and advanced packaging. In 2014 International 3D Systems Integration Conference $(3 D I C)$, pages $1-7$, Kinsdale, Ireland. IEEE.

IEC 61690-2:2000 (2000). Electronic design interchange format (EDIF) - Part 2: Version 400 . International Standard IEC 61690-2:2000, International Electrotechnical Commission, Geneva, $\mathrm{CH}$.

IEC 62402:2007 (2007). Obsolescence management - Application guide. International Standard IEC 62402:2007, International Electrotechnical Commission, Geneva, $\mathrm{CH}$.

IEEE Std 315-1975 (1975). Graphic Symbols for Electrical and Electronics Diagrams. Standard 315-1975, Institute of Electrical and Electronics Engineers.

Nagel, L. W. (1975). SPICE2: A Computer Program to Simulate Semiconductor Circuits. PhD Thesis, EECS Department, University of California, Berkeley, Berkeley, CA, USA.

Pelz, G. (1992). An interpreter for general netlist design rule checking. In Design Automation Conference, 1992. Proceedings., 29th ACM/IEEE, pages 305-310. IEEE Comput. Soc. Press.

Pröll, R., Rumpold, A., and Bauer, B. (2018). Applying Integrated Domain-Specific Modeling for Multi-concerns Development of Complex Systems. In Pires, L. F., Hammoudi, S., and Selic, B., editors, Model-Driven Engineering and Software Development, pages 247-271. Springer International Publishing.

Quarles, T., Newton, A. R., Pederson, D. O., and Sangiovanni-Vincentelli, A. (1993). SPICE3 Version $3 f 3$ User's Manual. Department of Electrical Engineering and Computer Science, University of California. Berkeley, CA.

Rumpold, A., Pröll, R., and Bauer, B. (2017). A Domain-aware Framework for Integrated Model-based System Analysis and Design. In 5th International Conference on Model-Driven Engineering and Software Development, pages 157-168. SciTePress.

Selic, B. (2003). The pragmatics of model-driven development. IEEE Software, 20(5):19-25. 